H I G H L I G H T S

\section{Target practice}

Some anti-angiogenic drugs have been found to only be effective in treating tumours at certain stages of development, indicating that its vasculature might change as the tumour develops. Erkki Ruoslahti, Doug Hanahan and colleagues have used a phage-display model to investigate these changes and to identify new therapeutic targets.

In two papers published in Cancer Cell, they report using a phage-display system to identify peptides that home to the blood vessels of different tumour types or at different stages of development or progression. Phage libraries can produce more than a billion random peptide sequences. The authors first incubated tumour cells, ex vivo, with phage libraries and isolated the bound phage. These phage were injected into mice for a round of in vivo selection, tumour-binding phage were again isolated and their peptide-encoding DNA inserts were sequenced.

In the first study, Hoffman et al. identified three peptides that specifically bound tumour vessels in a mouse model of epidermal carcinogenesis induced by human papillomavirus 16. One of these recognized the neovasculature in dysplastic skin but not in carcinomas. Its sequence was identical to that found in a loop of kallikrein-9, so it might bind to a substrate of this protease that is specifically expressed in the developing tumour vasculature. Further studies are required to determine

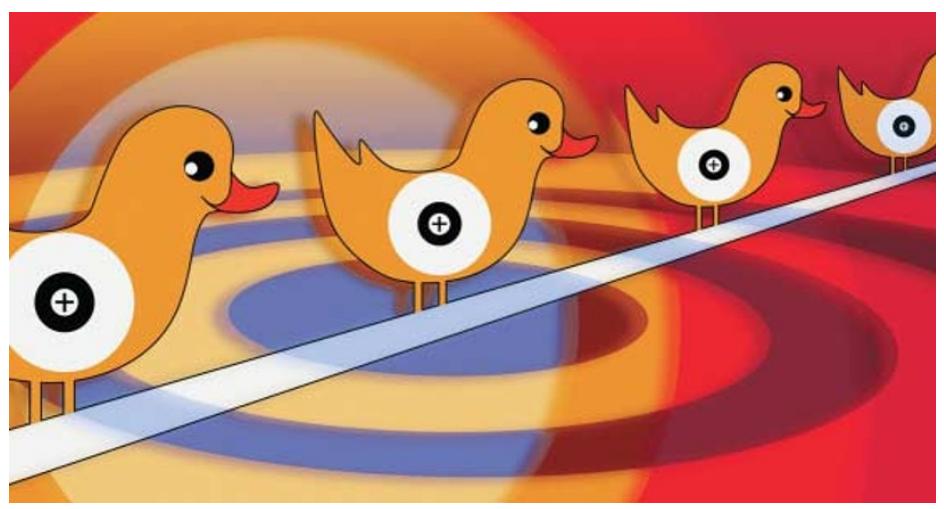

the role of this enzyme in tumour vasculogenesis.

In the second article, Joyce et al. searched for vascular markers that are specifically associated with tumours that form in RIP1-TAg2 mice, which are a model of pancreatic cancer. They characterized five peptides that selectively homed to neoplastic lesions in the pancreas, but not to islet $\beta$-cell tumours that were injected subcutaneously, to xenotransplant tumours from a human cancer cell line, or to squamous-cell tumours of the skin. Three of the peptides homed specifically to angiogenic islets and colocalized with markers that identify endothelial cells or pericytes. Investigation of candidate binding moieties revealed several proteins that have been implicated in some aspect of angiogenesis, including Pgdfs, Wnts, collagen XII, Fgfr1 and Tie1.

This approach can therefore not only be used to identify new proteins that are involved in tumour angiogenesis, but these peptides can also be used to target imaging agents or antiangiogeneic therapies specifically to tumour vessels.

Kristine Novak

(D) References and links ORIGINAL RESEARCH PAPER Hoffman, J. A. et al. Progressive vascular changes in a transgenic mouse model of squamous cell carcinoma. Cancer Cell 4, 383-392 (2003) | Joyce, J. A. et al. Stage-specific vascular markers revealed by phage display in a mouse model of pancreatic islet tumorigenesis. Cancer Cell 4, 393-403 (2003) FURTHER READING Ruoslahti, E. Specialization of the tumour vasculature. Nature Rev. Cancer $\mathbf{2}$, 83-90 (2002)
Results of a Phase III trial in patients with metastatic breast cancer show that ABI-007 (Abraxane; American Pharmaceutical Partners) - a solvent-free nanoparticle-coupled form of paclitaxel (Taxol; Bristol-Myers Squibb) — increased tumour response rate and slowed time to progression, compared with paclitaxel. Paclitaxel is one of the most effective anticancer agents, but dosing has been limited because of the toxic solvents that are included in the formulation. ABI-007 combines the paclitaxel with albumin into nanoparticles that are able to permeate microvessels and rapidly deliver higher doses of the drug to the tumour, but less to normal healthy cells. In a study of 454 patients, Joyce A.

O'Shaughnessy et al. reported that those who were given ABI-007 tolerated a higher dose of paclitaxel, experienced a lower incidence of neutropaenia, less severe forms of neuropathy and did not have the severe hypersensitivity reactions that are commonly associated with paclitaxel. Patients who received ABI-007 also had a significantly higher response rate than those who received paclitaxel (33\% versus 19\%) and had a longer time to tumour progression (21.9 weeks versus 16.1 weeks). These results also indicate that the solvent that is used to deliver paclitaxel, rather than the drug itself, might be responsible for many of the side effects. The lack of severe hypersensitivity reactions and the reduction in bone-marrow suppression in patients who were treated with ABI-007 might allow patients to avoid the costly medications that are necessary both before and after paclitaxel administration to reduce side effects.

ORIGINAL RESEARCH PAPER O'Shaughnessy, J. et al. ABI-007 (ABRAXANE) a nanoparticle albumin-bound (nab) paclitaxel, demonstrates superior efficacy vs Taxol in MBC: a Phase III trial. 26th Annual San Antonio Breast Cancer Symposium, abstract 44 (3-6 Dec 2003)

\section{Increasing sensitivity}

A Phase III trial has shown that the radiation sensitizer RSR13 (efaproxiral) improves the efficacy of whole-brain radiation therapy (WBRT) in patients with brain metastases. RSR13 is a synthetic small molecule developed by Allos Therapeutics, Inc that sensitizes hypoxic tumour tissues to radiation therapy by decreasing haemoglobin-oxygen binding affinity and, therefore, facilitates the release of oxygen in tumours. In a study of 538 women with breast cancer and brain metastases, Baldassarre Stea et al. found that including RSR13 in radiation therapy, along with supplemental oxygen, nearly doubled the median survival rate to 8.67 months, versus 4.57 months for patients who received radiation alone. Patients who were treated with RSR13 plus WBRT also had a higher tumour response rate than the control group (71.7\% versus $49.1 \%)$. A statistically significant stable or improvement in quality of life at 3 months post-treatment was observed in patients who received RSR13. Patients reported minimal serious adverse events - the most common side effect was hypoxaemia, which can be effectively managed with supplemental oxygen.

ORIGINAL RESEARCH PAPER Stea, B. et al. Results from a subgroup analysis of patients with metastatic breast cancer (MBC) in a phase 3, randomized, open-label, comparative study of standard whole brain radiation therapy (WBRT) with supplemental-oxygen, with or without RSR13, in patients with brain metastases. 26th Annual San Antonio Breast Cancer Symposium, abstract 175 (3-6 Dec 2003)

WEB SITE http://www.sabcs.org/ 